\title{
RULE OF RESCUE E ASSISTÊNCIA FARMACÊUTICA NO BRASIL: O CASO DAS DOENÇAS RARAS.
}

\author{
RULE OF RESCUE AND PHARMACEUTICAL ASSISTANCE IN BRAZIL: THE \\ CASE OF RARE DISEASES
}

\section{Voltaire de Freitas Michel}

Doutorado em Direito pela Universidade Federal do Rio Grande do Sul - UFRGS (2007), Mestrado em Direito - UFRGD (2003). Docente do Curso de Direito da IMED - Faculdade Meridional. Promotor de Justiça no Rio Grande do Sul.

\section{Sandra Regina Martini}

Doutorado em Evoluzione dei Sistemi Giuridici e Nuovi Diritti, Università Degli Studi di Lecce, UNILE, Itália, (2001). Mestrado em Educação pela Pontifícia Universidade Católica do Rio Grande do Sul - PUCRS, (1997). Especialização em Programação e Gerência de Sistemas de Saúde pela Escola Nacional de Saúde Pública - ENSP, (1987).

\section{Resumo}

O artigo explora o rule of rescue como um critério para decisões de incorporação de tecnologias em sistemas de saúde. O conceito de rule of rescue é importante na decisão de incorporação de medicamentos órfãos, destinados a doenças raras, casos em que o exame da custo-efetividade das drogas nos parâmetros mais restritos da medicina baseada em evidências, determina a sua rejeição. Propõem-se duas questões: em primeiro lugar, se o rule of rescue exerceu impacto na doutrina produzida no campo do direito sanitário no Brasil, a partir de uma revisão bibliográfica das fontes. Em segundo lugar, investiga-se se a rule of rescue exerceu influência nas recomendações de incorporação de medicamentos pela Conitec, sobretudo no campo das doenças raras, no período compreendido entre 2012 e 2018. Na primeira parte do trabalho, é apresentado 0 processo institucional de incorporação de medicamentos no Brasil e o tema da incorporação dos medicamentos órfãos. $\mathrm{Na}$ segunda parte, parte-se para as questões propostas. A metodologia empregada para avaliar o impacto doutrinário do princípio é a da revisão bibliográfica, focando nos periódicos especializados em direito sanitário no Brasil. Com relação ao segundo problema, buscou-se identificar as 
recomendações da Conitec que envolviam doenças raras, e, nesses relatórios, foram identificados, no tópico da discussão, a fundamentação da conclusão pela incorporação ou não, buscando identificar a presença do rule of rescue. As conclusões indicam que os fundamentos da incorporação e da não incorporação não levam em consideração nenhuma forma de rule of rescue, o que sinaliza para uma possibilidade de aperfeiçoamento desse processo.

Palavras-chave: Assistência Farmacêutica. Direito Sanitário. Doenças Raras. Medicamentos Órfãos. Rule of Rescue.

\begin{abstract}
The article explores the rule of rescue as a criterion for decisions to incorporate new technologies into public health systems. The concept of the rule of rescue is particularly important in the decision to incorporate orphan drugs for rare diseases, in which case the mere examination of the cost-effectiveness of drugs or the narrower evidence-based parameters of medicine rejection. For that, two questions are proposed: firstly, if the concept of rule of rescue had any impact on the doctrine produced in the field of sanitary law in Brazil, based on a bibliographical review of the most relevant sources. Second, it is investigated whether the rule of rescue had any influence on Conitec's recommendations to incorporate drugs, especially in the field of rare diseases, in the period between 2012 and 2018. In the first part of the paper, the process is presented of incorporation of drugs in Brazil and the topic of incorporation of orphan drugs. In the second part, we start with the questions initially proposed. The methodology used to evaluate the doctrinal impact of the principle is that of the bibliographic review, focusing on the specialized journals in sanitary law in Brazil. Regarding the second problem, the impact on the concrete decisions was aimed at identifying Conitec's recommendations involving rare diseases, and in those reports, the decisive rationale for the conclusion by the incorporation was identified in the discussion topic, seeking to identify the presence of the rule of rescue. The conclusions indicate that the fundamentals of incorporation and non-incorporation do not take into account any form of rule of rescue, which signals a possibility of perfecting this process.
\end{abstract}

Key-words: Health Law. Orphan Drugs. Pharmaceutical Assistance. Rare Diseases. Rule of Rescue. 


\section{CONSIDERAÇÕES INICIAIS}

A rule of rescue é um princípio bioético, segundo o qual, nos sistemas de saúde pública, deve ser dada prioridade aos que estão em situação de emergência, desconsiderando outros critérios fármaco-econômicos mais convergentes com a medicina baseada em evidências. O conceito de rule of rescue foi sugerido por Jonsen, em 1986 ${ }^{1}$, como um princípio deontológico, prescritivo de um dever, em contraposição a posições consequencialistas ou utilitaristas, que se inclinariam pela não incorporação, ou não fornecimento, de alguns tratamentos para pacientes em situação de emergência, segundo um cálculo de custo-efetividade. A ideia de uma rule of rescue é de particular interesse quando o que está em questão é a incorporação de medicamentos órfãos, destinados ao tratamento das doenças raras, cujos estudos, em geral, não atendem aos critérios estatísticos suficientes para amparar um cálculo de custo-efetividade.

O propósito desse artigo é responder a duas questões: em primeiro lugar, se o conceito de rule of rescue exerceu algum impacto na doutrina produzida no Brasil no campo do direito sanitário. Em segundo lugar, investigar se a rule of rescue influenciou, de alguma forma, nas recomendações de incorporação ou não incorporação de medicamentos pela Conitec, especificamente no campo das doenças raras, no período compreendido entre 2012 e 2018. Na primeira parte do trabalho, é apresentado o processo institucional de incorporação de medicamentos no Brasil e o tema da incorporação dos medicamentos órfãos relacionados com as doenças raras e, em seguida, uma conceituação do rule of rescue. Na segunda parte, parte-se para as questões propostas inicialmente, quais sejam, o impacto da rule of rescue na doutrina brasileira e nas recomendações da Conitec. A metodologia empregada para avaliar o impacto doutrinário do princípio é a da revisão bibliográfica, focando nos periódicos especializados em direito sanitário no Brasil. Com relação ao segundo problema, analisa-se o impacto nas recomendações, buscado identificar as que envolviam doenças raras. Em seguida, nas aludidas recomendações de incorporação ou não, foram identificadas, no tópico da discussão, a fundamentação determinante da recomendação, e se aludia a

\footnotetext{
${ }^{1}$ Neste artigo, Jonsen sugeriu o conceito de rule of rescue: JONSEN, Albert R. Bentham in a Box: technology assessment and health care allocation. The Journal of Law, Medicine \& Ethics, set. 1986. Disponível em: <http://journals.sagepub.com/doi/10.1111/j.1748-720X.1986.tb00974.x>. Acesso em: 12 jun. 2018.
} 
alguma forma de rule of rescue. Por fim, as fundamentações determinantes foram tabeladas, ano a ano.

\section{O PROBLEMA DA ASSISTÊNCIA FARMACÊUTICA EM DOENÇAS RARAS}

Nessa primeira parte do artigo, apresenta-se a questão das doenças raras, medicamentos órfãos e o desenho institucional da inclusão de novas tecnologias no Sistema Único de Saúde (SUS) no Brasil. Já ao final, apresenta-se o conceito de rule of rescue, com seus fundamentos filosóficos e desdobramentos jurídicos institucionais nos sistemas de saúde pública que o adotam.

\subsection{DOENÇAS RARAS E DOS MEDICAMENTOS ÓRFÃOS}

O conceito de "doenças raras" conheceu uma significativa alteração a partir dos anos 70. Até então, na literatura médica, "a raridade de uma doença era mencionada somente como uma categoria que o clínico devia ter em mente quando um paciente em seu consultório apresentasse um diagnóstico delicado" (HUYARD, 2009, p. 463). Na comunicação científica, as doenças raras eram descritas como relato de caso, geralmente resultante de apenas uma única observação clínica (WÄSTFELT; FADEEL; HENTER, 2006).

Esse conceito alterou-se para uma acepção mais precisa, que o relaciona diretamente com os medicamentos órfãos. Essa expressão surge nos Estados Unidos, a partir da publicação da Emenda Kefauver-Harris ou Emenda da Eficácia dos Medicamentos, de 1962. Essa emenda alterou, em parte, a Lei Federal de Alimentos, Medicamentos e Cosméticos, originalmente de 1938, como reação ao drama da talidomida, que provocara defeitos congênitos em milhares de crianças. A partir dessa emenda, os fabricantes de medicamentos viram-se obrigados a oferecer prova de eficácia e segurança de seus produtos, por meio de testes clínicos e a informar os efeitos colaterais na publicidade. Com tais exigências, alguns medicamentos deixaram de ser economicamente viáveis e foram abandonados pela indústria farmacêutica, daí o emprego da expressão "medicamentos órfãos". Segundo Llinares (2010), muitas razões levaram ao abandono dos medicamentos pela indústria farmacêutica: mercado limitado que não justificaria o investimento; 
número reduzido de pacientes para a realização de testes clínicos consistentes; falta de conhecimento sobre o mecanismo da doença; baixa conscientização da sociedade. Nesse revés, as doenças raras relacionaram-se com o conceito de medicamentos órfãos; ao final das contas, o conceito de raridade de uma doença vinculou-se à sua configuração econômica e não propriamente médica.

No plano epidemiológico, diferentes agências nacionais e internacionais empregam critérios variados para classificar uma doença nessa categoria. A União Europeia classifica como rara a doença que coloca em risco a vida do paciente, ou é cronicamente debilitante, e cuja prevalência é muito baixa (menos de 5 casos em cada 10 mil habitantes; Resolução o 141/2000, de 16/12/1999). No Japão, é considerada rara a doença que acomete quatro pessoas a cada 10 mil; nos Estados Unidos, é qualquer doença que afete menos de 200 mil pessoas no território nacional (OLIVEIRA, 2012). No Brasil, a Portaria o 199/2014, do Ministério da Saúde, considera doença rara aquela que afeta até 65 pessoas em cada 100.000 indivíduos, ou seja, 1,3 pessoas para cada 2.000 indivíduos.

Quando se leva em consideração a existência de um sistema público de saúde, cuja disponibilização de terapias e medicamentos está amparada em avaliações técnicas de saúde (ATS) e na medicina baseada em evidências (MBE), ambas desenhadas originalmente para doenças prevalentes, a posição dos medicamentos órfãos e doenças raras resulta enfraquecida, uma vez que "é bastante improvável que níveis convencionais de precisão estatística sejam prospectivamente obtidos nos ensaios clínicos habituais relacionados à avaliação de terapêuticas voltadas para as doenças raras" (OLIVEIRA, 2011). Além disso, "se procedimentos convencionais de avaliações de tecnologia em saúde (ATS) forem aplicados a estes medicamentos, muito provavelmente nenhum deles será custoefetivo" (OLIVEIRA, 2012).

A propósito, os últimos 30 anos têm testemunhado o crescente emprego das avaliações de tecnologias em saúde (ATS) como instrumento de tomada de decisão acerca da sua incorporação a sistemas públicos de saúde. A despeito de sua utilidade na gestão e aplicação de recursos financeiros escassos, as técnicas de ATS podem se revelar inadequadas quando empregadas na definição de políticas de assistência farmacêutica e provisão de medicamentos a portadores de doenças 
raras $^{2}$. Ao longo dos anos, evidências nesse sentido vêm se acumulando em todo o mundo. Tendo como regra a recomendação de que somente as tecnologias com resultados consistentes e robustos devem ser incorporadas aos sistemas de saúde, a ATS é um método de síntese do conhecimento científico para a tomada de decisões, do ponto de vista da eficácia, da efetividade e dos custos relacionados às implicações da utilização das tecnologias em saúde (NITA; NOBRE; COSTA, 2010). Avaliações técnicas em saúde têm sido crescentemente empregadas para informar decisões acerca da cobertura de medicamentos para doenças prevalentes ou raras neste ou naquele país, visando a assegurar que escolhas racionais e baseadas em evidências sejam feitas no contexto da administração de recursos escassos, como aqueles conceitualmente disponíveis para a saúde. No entanto, um work package inscrito no projeto Advance-HTA que, financiado pela União Europeia, sistematicamente investigou processos de tomadas de decisão em ATS envolvendo dez medicamentos órfãos em quatro países, produziu conclusões surpreendentes, que se tornaram canônicas na literatura científica sobre o tema (ADVANCE-HTA, 2015).

O referido estudo verificou uma variedade de questões, que comissões de ATS têm de enfrentar, quando lidam com decisões envolvendo a incorporação de medicamentos órfãos a seus sistemas de saúde. Assim, quando avaliações econômicas são empregadas em ATS, os medicamentos órfãos frequentemente falham em observar critérios-padrão de custo-efetividade, devido a seus altos custos de aquisição e o alto grau de incerteza da base de evidências.

Nesse cenário, verifica-se que o conceito de doenças raras e medicamentos órfãos encontra-se no cruzamento de vários interesses contrapostos, que nem sempre serão exclusivamente médicos ou altruístas: de um lado, o interesse da indústria farmacêutica em concentrar seus esforços intelectuais e produtivos na ponta certa dos medicamentos consagrados; de outro, a necessidade médica que se depara com doenças de pouca prevalência, o que dificulta a comprovação da "evidência" clínica da eficácia de uma certa droga; interligando os interesses, a

\footnotetext{
2 Sugerindo que a medicina baseada em evidências, ou avaliação de tecnologias em saúde, poderia representar um fator determinante para as decisões judiciais, DIAS, Eduardo Rocha; SILVA JÚNIOR, Geraldo Bezerra da. A medicina baseada em evidências na jurisprudência relativa ao direito à saúde. Revista Einstein, v. 14, n. 1, jan./mar. 2016, pp. 1-5. No mesmo sentido, porém ressalvando, em nota, o caso especial das doenças raras, DUARTE, Clarice Seixas; BRAGA, Paulo Vitor Bérgamo. A utilização dos protocolos clínicos e diretrizes terapêuticas e a racionalização da judicialização do direito à saúde. Revista de Direito Sanitário, v. 18, n. 1, pp. 171-190. São Paulo, mar/jun. 2017.
} 
tentativa do Poder Público de conferir certa racionalidade aos seus gastos com a assistência terapêutica e farmacêutica, não gastando com medicamentos meramente experimentais; e, por fim, talvez a ponta mais fraca, o paciente, que certamente necessita da droga experimental, porém, também, pode ver-se iludido por uma promessa que não pode ser cumprida. $O$ caso das doenças ultrarraras, que no Brasil são consideraras as que têm incidência inferior a 1 caso para cada 50.000 habitantes, suscita ainda mais controvérsia (HUGHES; TUNNAGE; YEO, 2005).

\subsection{ASSISTÊNCIA TERAPÊUTICA E FARMACÊUTICA, INCLUSÃO DE NOVAS TECNOLOGIAS NO SUS (SISTEMA ÚNICO DE SAÚDE) E DOENÇAS RARAS NO BRASIL}

Um dos aspectos mais importantes do Sistema Único de Saúde é a assistência terapêutica integral, inclusive farmacêutica, conforme preceitua 0 art. 6 , inciso I, alínea 'd', da Lei $n^{\circ} 8.080$, de 1990, que estruturou o SUS no país, em atenção ao art. 196 da Constituição Federal de 1988. Recentemente, em 2011, a Lei do Sistema Único de Saúde foi alterada, em parte, no que diz respeito à assistência terapêutica, incluindo novas regras para a inclusão de novas tecnologias ao sistema (alteração promovida pela Lei no 12.401/2011).

Segundo a Lei do SUS, a assistência terapêutica integral compreende, sobretudo, a dispensação de medicamentos e produtos de interesse para a saúde, cuja prescrição esteja em conformidade com as diretrizes terapêuticas definidas em protocolo clínico, em conformidade com o disposto no art. 19-P da Lei do SUS.

No parágrafo único do artigo 19-O, esclarece-se que, em qualquer hipótese, "medicamentos ou produtos de que trata o caput deste artigo serão aqueles avaliados quanto à sua eficácia, segurança, efetividade e custo-efetividade para as diferentes fases evolutivas da doença ou do agravo à saúde de que trata o protocolo" (BRASIL, 2011, documento online).

E, essa incorporação, como expõe o artigo 19-Q, § 2º, incisos I e II da referida lei, levará em consideração, necessariamente "as evidências científicas sobre a eficácia, a acurácia, a efetividade e a segurança do medicamento, produto ou procedimento objeto do processo, acatadas pelo órgão competente para o registro ou a autorização de uso" e "a avaliação econômica comparativa dos benefícios e dos custos em relação às tecnologias já incorporadas, inclusive no que se refere aos 
atendimentos domiciliar, ambulatorial ou hospitalar, quando cabível" (BRASIL, 2011, documento online).

No que toca ao caso das doenças raras e os medicamentos órfãos, o art. 19T é o mais elucidativo e pertinente. Segundo esse dispositivo legal, "são vedados, em todas as esferas de gestão do SUS", "[...] o pagamento, o ressarcimento ou o reembolso de medicamento, produto e procedimento clínico ou cirúrgico experimental, ou de uso não autorizado pela Agência Nacional de Vigilância Sanitária - Anvisa" e "[...] a dispensação, o pagamento, o ressarcimento ou o reembolso de medicamento e produto, nacional ou importado, sem registro na Anvisa" (BRASIL, 2011, documento online).

Como já destacado anteriormente, de certa forma, a avaliação das tecnologias em saúde, embora necessária para a racionalização dos recursos públicos, eventualmente produz o efeito indesejado de ignorar medicamentos fundamentais para uma parcela importante da população. A aplicação ortodoxa de procedimentos de ATS e a desconsideração de elementos ad hoc em tais deliberações poderia explicar parte substancial da judicialização da saúde no país. Com efeito, entre os medicamentos mais buscados por meio de ações judiciais são indicados para doenças raras (SOUZA, 2017, documento online). Assim, tal viés metodológico, acima descrito, poderia estar comprometendo os princípios da equidade, universalidade e integralidade que norteiam o SUS, no que se refere à assistência farmacêutica a portadores de doenças raras.

Conforme a estrita literalidade da Lei do SUS, tais medicamentos não deveriam ser fornecidos, porque Ihes falta o registro na Anvisa; no entanto, na via judicial, a interpretação da legislação infraconstitucional, polarizada pelo texto constitucional, tem permitido a alguns pacientes obtê-lo.

\subsection{RULE OF RESCUE: FUNDAMENTOS FILOSÓFICOS E DOGMÁTICOS}

Há que se distinguir entre os fundamentos filosóficos de uma rule of rescue e sua expressão jurídico-dogmática, que pode adotar diferentes formatos, conforme o compasso histórico e as diferentes decisões políticas.

\subsubsection{Fundamentos filosóficos}


Segundo Jonsen (1986), formulador inicial do princípio da rule of rescue no campo dos sistemas de saúde, os cálculos de custo-efetividade levados a efeito pelas instâncias burocráticas representariam o sonho de Jeremy Bentham, pai da corrente filosófica do utilitarismo. Para Jonsen (1986), esses formuladores de políticas públicas de saúde não são, propriamente, teóricos utilitaristas, visto que suas referências não provêm dos ensinamentos de Bentham, Mill ou Sidgewick. No entanto, se observado o lado prático, há um viés utilitarista na sua ação, pois, a "questão é se uma tecnologia em particular pode quantitativamente demonstrar um efeito mais positivo se em comparação com outras tecnologias" (JONSEN, 1986, documento online).

O autor dá a essa barreira o nome de rule of rescue. Conforme Jonsen (1986), a resposta à iminência da morte exige um resgate ao condenado. "Nós jogamos uma corda para quem está se afogando, corremos em direção a prédios em chamas para resgatar os presos, despachamos times de resgate para localizar os atingidos por avalanches" (JONSEN, 1986, documento online). Há uma similaridade entre a moralidade do resgate e o tratamento médico, visto que as cordas seriam corações artificiais, as corridas equivaleriam às UTIs móveis e, os times, se equiparariam aos grupos de transplante. "O imperativo de resgatar é, sem dúvida, de grande importância moral; mas o imperativo parece crescer em direção a uma compulsão, mais instintiva que racional" (JONSEN, 1986, documento online).

Apesar da familiaridade de Jonsen (1986) com filósofos que se classificariam como deontológicos, que adotariam máximas de comportamento sem levar em consideração as consequências, o autor surpreende-se por nunca haver se deparado com tais princípios na prática, a não ser em processos de avaliação de tecnologias para o salvamento de vidas.

A lógica parecia conduzir à conclusão de que a tecnologia não era custoefetiva. Antes que a conclusão pudesse ser tomada, no entanto, a rule $o$ rescue apresentou-se como uma barreira intransponível. A conclusão lógica sobre a avaliação cedeu espaço, e a tecnologia, empregada com precaução, evidentemente, saiu-se vencedora. A diálise renal era a vencedora nos anos 60; recentemente, os transplantes de órgãos vitais determinaram a criação de uma força-tarefa federal (JONSEN, 1986, documento online).

Em certo sentido, a rule of rescue representa, na bioética e no direito sanitário, a aplicação de um princípio de justiça prioritarista, em contraste com as 
opções utilitaristas ou igualitaristas. A respeito das implicações morais do contraste entre a rule of rescue e as posições utilitaristas, Mckie e Richardson (2003) explicam que a rule of rescue representa um conjunto de características. Essencialmente, demonstraria um imperativo psicológico, uma necessidade de se resgatar alguém (ou alguns) que esteja na iminência da morte ou de um grave dano. Os autores traduzem essa reação como um "choque de horror" que orienta os indivíduos, visto que não há tempo hábil para assimilar os acontecimentos enquanto eles ocorrem em tempo real.

Se a ameaça é para a própria vida, ou apenas para o bem-estar da vítima, normalmente há uma alta probabilidade de ocorrer (se as medidas de resgate não forem realizadas), e isso ocorrerá no curto prazo, e não no longo prazo. Mais visivelmente, o RR está em desacordo com os meios de avaliação convencionais: há pouco tempo e pouca oportunidade para refletir sobre o custo das medidas de resgate tomadas quando um indivíduo identificável está enfrentando uma ameaça real de morte evitável (ou outro dano grave). (MCKIE; RICHARDSON, 2003, p. 2.410). ${ }^{3}$

Segundo Jeremy Waldron (2001), a teoria utilitarista é um dos exemplos de teoria da justiça polarizada pela ideia de bem, ainda que a ser alcançado por meio de um cálculo interessado. Em síntese, é moral, e da mesma forma, justa, a decisão que produzir um melhor resultado, que aproximar a situação de fato de um objetivo declarado. O foco da moralidade utilitária está nas consequências da ação, em vista do objetivo almejado, e não na ação em si, que pode até mesmo ser moralmente condenável por outros critérios de apreciação. ${ }^{4}$

Para ilustrar a lógica, Thomas Nagel (1979 apud PARFIT, 1997) inicia uma reflexão a respeito da utilidade de uma ação a partir do exemplo dos dois filhos, que pode ser assim sumarizado: um casal tem dois filhos, um saudável e outro que

\footnotetext{
${ }^{3}$ Whether the threat is to life itself, or merely to the well-being of the victim, there is typically a high probability of it occurring (if rescue measures are not undertaken), and it will occur in the short-term rather than the long-term. Most conspicuously, the RR is at odds with conventional CEA: there is little time, and little inclination, to reflect on the cost of the rescue measures undertaken when an identifiable individual is facing a real threat of avoidable death (or other serious harm). MCKIE, John; RICHARDSON, Jeff R. J. The rule of rescue. Social Science and Medicine, v. 56, ano 12, pp. 24072419, jul. 2003. Disponível em: <https://research.monash.edu/ en/publications/the-rule-of-rescue>. Acesso em: 01 jul. 2018.

4 A respeito dos argumentos pró e contra o utilitarismo, ver SMART, J.C.C.; WILLIAMS, B. Utilitarianism: for \& against. Cambridge: Cambridge University Press, 1973; KYMLICKA, Will. Filosofia política contemporânea. São Paulo: Martins Fontes, 2006; NAGEL, Thomas. Mortal question. Cambridge: Cambridge University Press, 1979, pp. 123-124, apud PARFIT, Derek. Equality and priority. v. 10, n. 3. Oxford: Blackwekk Publishers, 1997. pp. 201-221. Disponível em: $<$ http://individual.utoronto. ca/stafforini/parfit/parfit_-_equality_and_priority.pdf>. Acesso em: 13 jun. 2018.
} 
precisa de atenção médica. O dilema é: mudar-se para um subúrbio, onde o saudável poderá brincar na rua e ter uma vida melhor, ou para a cidade, onde morarão enclausurados num pequeno apartamento, porém mais próximos do atendimento médico para o filho doente.

Derek Parfit (1977) sintetiza o problema inicial com o seguinte esquema, adaptado para as finalidades deste trabalho:

\section{TABELA 1 - Problema de Parfit (primeiro cenário)}

\begin{tabular}{llc}
\hline & Criança saudável & Criança doente \\
\hline Mudar para a cidade & $\begin{array}{l}20 \text { (nível de benefício } \\
\text { alcançado ou de } \\
\text { felicidade) }\end{array}$ & 10 \\
Mudar para o subúrbio & 25 & 9 \\
\hline
\end{tabular}

Fonte: Dereck Parfit (1977).

Segundo Derek Parfit (1977), um utilitarista escolheria, entre as duas soluções, a mudança para o subúrbio, porque ali o menino saudável seria muito mais feliz (índice 25), e o menino doente, apenas um pouco mais triste (índice 9), totalizando um índice de felicidade de 34. A mudança para a cidade, que tornaria o menino doente um pouco mais feliz (índice 10), não faria o menino saudável muito feliz, alcançando o índice de 30 na felicidade combinada de ambos. Nesse cenário, o moral e justo seria a opção "subúrbio", não obstante a crueldade com o menino doente.

Em contraposição ao critério utilitário, o critério igualitário, o justo ou o desejável, é um resultado em que todos tenham acesso ao mesmo serviço. O ideal a ser alcançado é a igualdade na distribuição dos bens. Num molde mais radical, segundo este critério, qualquer desigualdade é condenável e exprime uma situação pior, sob o ponto de vista da justiça, que um cenário de total igualdade.

Segundo Derek Parfit (1977), um igualitário reputaria mais justa uma melhor distribuição, ainda que os benefícios globalmente sejam menores. Voltando ao exemplo de Thomas Nagel, a respeito dos meninos, eis a tabela novamente:

TABELA 2 - Problema de Parfit (segundo cenário)

\begin{tabular}{llc}
\hline & Criança saudável & Criança doente \\
\hline Mudar para a cidade & $\begin{array}{l}20 \text { (nível de benefício } \\
\text { alcançado) }\end{array}$ & 10 \\
\hline
\end{tabular}




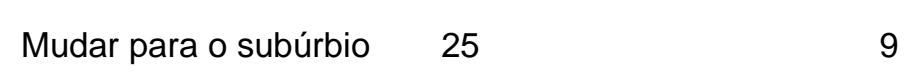

Fonte: Dereck Parfit (1977).

Um igualitarista escolheria a primeira linha, pois no cenário de 20/10 há mais igualdade do que no cenário $25 / 9$, ainda que, no primeiro cenário, o menino doente tenha sido prejudicado novamente.

Segundo Derek Parfit (1977), as razões de Nagel em favor da opção "mudar para a cidade" são, na verdade, polarizadas pela ideia de prioridade: quer dizer, antes de atender o supérfluo ou o abundante, a justiça determinaria que se satisfizesse as necessidades imediatas dos mais prejudicados. Tal o sentido do prioritarianismo, conforme Parfit (1977): beneficiar com prioridade as pessoas que têm mais necessidade.

A posição prioritária, no entanto, enfrenta objeções. A primeira delas é a da ineficiência. Segundo esse argumento, a solução da prioridade pode ser ineficiente, como ilustra o caso do tratamento conferido aos soldados feridos no campo de batalha e qual a extensão do tratamento médico que lhes deve destinar. Como o exemplo citado por Wolf e De-Shalit (2007), os soldados feridos podem ser classificados em três categorias: a) o que vai morrer ou é muito custoso salvar; b) o que com pouca ajuda médica pode ser salvo e voltar para a linha de batalha: c) 0 que está praticamente bem, só precisa de atendimento médico mínimo. Segundo um argumento baseado no utilitarismo, polarizado pelo interesse de vencer a batalha, só faria sentido gastar com "b". Seria um desperdício gastar com "a", assim como com "c". Conforme o argumento prioritário, faz sentido gastar com o "a", porque tem a maior necessidade; essa seria, no entanto, uma decisão ineficiente sob o ponto de vista da vitória na batalha (WOLFF; DE-SHALIT, 2007).

Conforme Wolf e De-Shalit (2007), este argumento contrário ao prioritarianismo pode ser refutado. Segundo eles, a prioridade tem um aspecto moral, e o argumento da ineficiência é limitado ao aspecto econômico e se prende ao cenário mais amplo; na verdade, o pouco que se pode fazer pelo muito necessitado faz grande diferença.

Ainda no campo da prioridade, reforça essa ideia a concepção de Amartya Sen, exposta por Martha Nussbaum (2011) em sua teoria das capacidades e das necessidades humanas básicas. Para Sen (1999), as capacidades e necessidades 
humanas básicas servem como índice de comparar o bem-estar entre diversos países (quais e em que intensidade as capacidades podem ser desenvolvidas num certo país). Conforme Nussbaum (2011), a abordagem de Sen é superior aos utilitaristas e rawlsianos, não tendo apenas uma função econométrica, mas de estabelecer uma teoria política, uma teoria sobre justiça social. Segundo Martha Nussbaum (2011), cuja teoria política é inspirada na obra de Sen, as capacidades representam o que uma pessoa é capaz de fazer ou de ser, em certo aspecto da vida. É um conjunto de oportunidades ou liberdades substanciais; é pluralista a respeito dos valores. Nussbaum leva adiante esse conceito de Sen, estipulando uma lista das capacidades centrais.

Tanto para Nussbaum quanto Sen, as capacidades não são redutíveis, de modo que a falta de uma possa ser compensada por outra. Há, no entanto, situações trágicas em que o agente tem que optar por funcionar uma ou outra capability (ex: ter que parar de estudar para cuidar dos filhos). Segundo Sen, não tem como fazer uma lista de preferências, embora Nussbaum entenda em alguns casos é evidente qual é a melhor solução.

De acordo com uma teoria política polarizada por essa ideia de capacidades, é papel das instituições implementar um mínimo de condições, e elas podem decorrer do direito constitucional ou dos seus princípios básicos. Em certo sentido, o conceito de rule of rescue seria compatível com a visão de Sen e Nussbaum a respeito do atendimento de necessidades básicas.

\subsubsection{Expressão jurídico-dogmática}

Tendo em vista que a rule of rescue representa uma opção prioritarista, verifica-se que dogmaticamente foi acolhida em dois sistemas públicos de saúde, expressamente, o do Reino Unido e da Austrália.

No Reino Unido, o órgão central deliberativo a respeito de incorporação de novas tecnologias é o Nice, que é integrado por um Conselho de Cidadãos, a quem compete opinar a respeito de questões de valor social no sistema de saúde (COOKSON; MCCABE; TSUCHIYA, 2007). Um relatório recente do Nice tratou especificamente da rule of rescue ${ }^{5}$. Aos membros desse conselho foram

\footnotetext{
${ }^{5}$ Relatório do Nice abordando rule of rescue: NATIONAL INSTITUTE FOR HEALTH AND CLINICAL EXCELLENCE. Citizens council report: the rule of rescue. Nice: London, 2006. Disponível em:
} 
apresentadas as seguintes questões:

1. Há uma preferência por salvar pessoas em risco de vida ao invés de melhorar as condições de saúde de pessoas cujas vidas não estão em iminente risco?

Ou

2. salvar a vida de muitas pessoas no futuro por meio de programas de prevenção (tais como tratando pressão alta ou níveis altos de colesterol)? Se o conselho entende que o Nice deve ignorar a rule o rescue - por quê? Se o conselho entende que o Nice deve considerar a rule of rescue - em que circunstâncias e quais os limites? (NICE, 2006, documento online).

No final da deliberação, o conselho entendeu, por maioria, que o Nice deveria levar em consideração a rule of rescue e definir os seus limites. O resultado da deliberação do conselho foi o seguinte check list:

1. a intervenção é necessária para evitar a imediata perda de uma vida?

2. há uma boa chance de aumentar a expectativa de vida?

3. resultará numa significante melhora na qualidade de vida? Os efeitos colaterais do tratamento são graves e poderiam não compensar o benefício do tratamento?

4. quais as consequências da ausência do tratamento?

5. quais são os tratamentos alternativos e como se comparam com o proposto?

6. há prováveis ganhos médicos futuros relacionados com a pesquisa provocada pelo tratamento?

7. os custos são proibitivos para o sistema nacional de saúde? Em que medida ele aumenta o custo ao sistema nacional de saúde e à sociedade como um todo?

8. em que medida é demonstrada a custo efetividade.

9. há boas razões para acreditar que estabeleceria um precedente para outros grupos de pacientes que postulam por tratamentos menos custo-efetivos?

10. evitaria um risco à saúde coletiva, como por exemplo, uma ameaça de epidemia?

11. as pessoas sentiriam que o valor da sociedade seria diminuído se parecesse desumano ignorar a rule of rescue?

<https://www.nice.org.uk/Media/Default/Get-involved/Citizens-Council/Reports/CCReport06RuleOf Rescue.pdf>. Acesso em: 14 jun. 2018. 
$\mathrm{Na}$ Austrália, a mesma deliberação esteve sob a responsabilidade do Australian Phamaceutical Benefits Advisory Committee (APBAC), que especificou três requisitos para a aplicação do rule of rescue na assistência farmacêutica nacional:

a) não há alternativa na Austrália para tratar pacientes com a condição médica restrita. Esse é um requisito absoluto. Significa que não há intervenção farmacológica ou não-farmacológica para esses pacientes.

b) a condição médica definida pela restrição é severa, progressiva e presumivelmente conduzirá a uma morte prematura. Quanto mais severa a condição, ou quanto mais cedo a pessoa afetada poderá morrer, ou quanto mais perto essa pessoa está da morte, mais decisivamente a rule of rescue deverá ser levada em consideração pelo comitê.

c) a condição médica definida por essa restrição aplica-se apenas a um número muito pequeno de pacientes. Novamente, quanto menos pacientes, maior influência deverá ter a rule of rescue em consideração pelo comitê. No entanto, o comitê está consciente que o sistema público de saúde é baseado na comunidade e não pode atender a casos específicos.

O contraste entre as duas soluções dogmáticas-jurídicas encontradas pelos dois sistemas, o inglês e o americano, conduz à conclusão de que, a partir de um conceito genérico, decorrente de uma percepção da justiça como estabelecimento de uma prioridade, podem ser desdobradas soluções institucionais em grande parte divergentes e, até mesmo, contraditórias. Basta perceber, por exemplo, que o check list do Conselho de Cidadãos do Nice é expresso ao incluir, dentre os requisitos, sem estabelecer uma prioridade, a análise da custo-efetividade; nos critérios empregados na Austrália, no entanto, tal variável não é levada em consideração, acentuando-se $o$ aspecto da ausência de outro tratamento.

Prospectivamente, no caso de aplicação do rule of rescue pela Conitec, o que se antecipa é a necessidade de desdobramento do princípio bioético e de justiça em regras determinadas, que permitam justificar a sua aplicação em casos concretos.

\section{O IMPACTO DO PRINCÍPIO DA RULE OF RESCUE NO DIREITO BRASILEIRO}

Nessa parte do trabalho, avalia-se o impacto que o conceito de rule of rescue 
teve no direito sanitário brasileiro até os dias atuais e, em seguida, se foi, em algum momento, considerado nas recomendações da Conitec.

\subsection{NA DOUTRINA NACIONAL}

Para avaliar o impacto do princípio do rule of rescue na doutrina nacional, empregou-se a seguinte técnica: preliminarmente, na Plataforma Sucupira ${ }^{6}$, foram identificados os periódicos nacionais que contivessem em sua denominação a expressão "direito sanitário". Em seguida, nos mecanismos de busca de cada um desses periódicos, buscou-se as seguintes palavras-chave: "rule of rescue"; "regra de resgate"; Conitec. A partir daí os trabalhos publicados foram analisados, com o descarte das resenhas e comentários jurisprudenciais. Nos trabalhos identificados, buscou-se, no seu texto, alguma referência a rule of rescue ou regra de resgate; em seguida, verificou-se, nos seus textos, se havia alguma menção a regras de exceção nos processos de incorporação de medicamentos.

$\mathrm{Na}$ primeira etapa, foram identificados apenas dois periódicos na área de direito sanitário, a Revista de Direito Sanitário ${ }^{7}$ e os Cadernos Ibero-Americanos de Direito Sanitário". Com as palavras-chave "rule of rescue" ou "regra de resgate", não foram encontrados trabalhos. Com a indexação "Conitec" foram identificados, entre

\footnotetext{
6 "É uma nova e importante ferramenta para coletar informações, realizar análises e avaliações e ser a base de referência do Sistema Nacional de Pós-Graduação (SNPG). A Plataforma deve disponibilizar em tempo real e com muito mais transparência as informações, processos e procedimentos que a Capes realiza no SNPG para toda a comunidade acadêmica. Igualmente, a Plataforma propiciará a parte gerencial-operacional de todos os processos e permitirá maior participação das pró-reitorias e coordenadores de programas de pós-graduação. A escolha do nome é uma homenagem ao professor Newton Sucupira, autor do Parecer № 977 de 1965. O documento conceituou, formatou e institucionalizou a pós-graduação brasileira nos moldes como é até os dias de hoje". BRASIL. Ministério da Educação. Plataforma Sucupira. 01 abr. 2014. Disponível em: <http://www.capes.gov.br/avaliacao/plataforma-sucupira>. Acesso em: 14 jun. 2018.

7 Publicada pela USP, "a Revista de Direito Sanitário tem por missão divulgar o conhecimento e fomentar discussões no campo interdisciplinar do Direito Sanitário, independentemente da formação original do autor". Sua qualificação é A2 e a periodicidade é quadrimestral. UNIVERSIDADE DE SÃO PAULO. Revista de Direito Sanitário. São Paulo. Disponível em: <https://www.revistas.usp.br/rdisan/about>. Acesso em: 14 jun. 2018.

8 "Cadernos Ibero-Americanos de Direito Sanitário/Cuadernos Iberoamericanos de Derecho Sanitario / Iberoamerican Journal of Health Law é uma publicação trilíngue (português, espanhol e inglês), trimestral, de acesso livre, destinada a difundir a produção acadêmica no campo do Direito Sanitário. É uma publicação do Programa de Direito Sanitário da Fundação Oswaldo Cruz Brasília, dirigida a professores, pesquisadores e estudantes de Direito, de Ciências da Saúde e de Ciências Sociais; operadores do Direito; profissionais de saúde e gestores de serviços e sistemas de saúde." FUNDAÇÃO OSWALDO CRUZ. Cadernos Ibero-Americanos de Direito Sanitário. Brasília-DF. Disponível em: <https://www.cadernos.prodisa.fiocruz.br/index.php/cadernos/about/editorialPolicies\#focusAndScope> . Acesso em: 14 jun. 2018.
} 
2013 e 2017, dez trabalhos acadêmicos, podendo-se perceber um aumento do interesse na matéria no ano de 2017, quando foram publicados seis.

Dentre esses trabalhos, em nenhum deles há menção a uma rule of rescue ou regra de resgate. Apenas quatro tratavam especificamente da atuação da Conitec, direta ou indiretamente e, na sua totalidade, tais trabalhos transitam na necessidade de estabelecimento de regras gerais, de "racionalização" nos sistemas de saúde. Dentre os trabalhos consultados, um conclui que "com a judicialização da saúde as aquisições são realizadas sem planejamento ou estabelecimento de critérios mínimos o que pode comprometer a sustentabilidade do SUS" (NOGUEIRA; CAMARGO, 2017, pp. 120); outro, no sentido de que

[...] o processo de incorporação de medicamentos no SUS necessita de maior participação social, principalmente dos usuários e que conhecimento técnico especializado nas avaliações de tecnologias não devem se constituir numa justificativa para a não inserção da sociedade nesse processo (GOMES, 2015, p.120).

E, um terceiro artigo sugere a aplicação dos critérios da Conitec para as decisões judiciais (CIARLINI, 2015); e, por fim, o quarto artigo também sugere a adoção dos Protocolos Clínicos e Diretrizes Terapêuticas (PCDTs) como parâmetro objetivo a ser utilizado pelos tribunais nas ações judiciais, cujo objeto são prestações de naturezas positivas voltadas à efetivação do direito à saúde, embora com a ressalva de que não se trata de um parâmetro absoluto, podendo o conteúdo do PCDT ser questionado, até mesmo judicialmente (DUARTE, 2017). Não se encontrou, no entanto, menções expressas ao rule of rescue nas fontes consultadas no direito brasileiro.

\subsection{NAS RECOMENDAÇÕES DA CONITES}

A Comissão Nacional de Incorporação de Tecnologias ao SUS (Conitec) foi criada pela Lei no 12.401 , de 28 de abril de 2011, que modificou, em parte, a Lei no 8.080/90, a Lei do Sistema Único de Saúde. A nova comissão sucedeu a Comissão de Incorporação de Tecnologias (Citec), instituída em 2006, e tem por objetivo assessorar o Ministério da Saúde - MS nas atribuições relativas à incorporação, exclusão ou alteração de tecnologias em saúde pelo SUS, bem como na 
constituição ou alteração de Protocolos Clínicos e Diretrizes Terapêuticas (PCDT). A comissão foi regulamentada pelo Decreto $n^{\circ}$ 7.646, de 21 de dezembro de 2011.

Conforme informações obtidas no próprio site da Conitec, as alterações institucionais mais destacadas na transição da Citec para a Conitec foram as seguintes: ampliação dos membros, de 5 titulares e 5 suplentes, para 13 titulares, cada um com dois suplentes, representando diferentes órgãos públicos e privados; exigência de uma documentação mínima, dentre a qual se inclui evidência científica que demonstre que a tecnologia pautada é, no mínimo, tão eficaz e segura quanto aquelas disponíveis no SUS para determinada indicação e estudo de avaliação econômica comparando com as tecnologias já disponibilizadas no SUS; submissão à consulta púbica em todos os casos; possibilidade de realização de audiência pública sobre temas em avaliação, a critério do Secretário de Ciência, Tecnologia e Insumos Estratégicos do Ministério da Saúde, caso julgue necessário; prazo máximo para avaliação de processo pela Conitec não deverá ser superior a 180 (cento e oitenta) dias, contado da data em que foi protocolizado o pedido, admitida prorrogação por 90 (noventa) dias corridos, quando as circunstâncias exigirem; a partir da publicação da decisão de incorporar medicamento, produto ou procedimento, ou mesmo protocolo clínico e diretriz terapêutica, as áreas técnicas do MS têm prazo máximo de 180 (cento e oitenta) dias para efetivar a oferta ao SUS.

A respeito da composição da Conitec, a norma regulamentadora optou por uma conformação predominantemente pública; dos seus treze membros, apenas o representante do Conselho Nacional de Saúde procede da sociedade civil; não há assento para associação de pacientes ou fabricantes de medicamentos ou, até mesmo, à comunidade médica.

Sobre a iniciativa para a instauração do processo administrativo de incorporação, o decreto regulamentador da Conitec não aponta os legitimados, porém ressalva que, em se tratando do próprio Ministério da Saúde, "serão consideradas as informações disponíveis e os estudos técnicos já realizados para fins de análise pela Conitec" ( $\$ 4^{\circ}$ do art. 15) (BRASIL, 2011, documento online).

Embora aparentemente o regulamento prescreva a obrigatoriedade das consultas públicas, as audiências públicas não são compulsórias, que poderão ser determinadas pelo Secretário de Ciência, Tecnologia e Insumos Estratégicos do Ministério da Saúde, conforme a relevância da matéria (art. 21) (BRASIL, 2011). 
Para o fim de recomendar a respeito da incorporação de uma nova tecnologia, a Conitec deverá levar em consideração (art. 18, da Lei do SUS):

I - as evidências científicas sobre a eficácia, a acurácia, a efetividade e a segurança do medicamento, produto ou procedimento objeto do processo, acatadas pelo órgão competente para o registro ou a autorização de uso; II - a avaliação econômica comparativa dos benefícios e dos custos em relação às tecnologias já incorporadas, inclusive no que se refere aos atendimentos domiciliar, ambulatorial ou hospitalar, quando cabível; e III - o impacto da incorporação da tecnologia no SUS (BRASIL, 2011, documento online).

A decisão final, após consulta pública e, eventualmente, audiência pública, caberá ao Secretário de Ciência, Tecnologia e Insumos Estratégicos do Ministério da Saúde, com recurso, sem efeito suspensivo, no prazo de dez dias, contados a partir da ciência ou divulgação oficial da decisão, dirigido ao próprio Secretário; mantida a decisão, ele mesmo encaminhará de ofício o recurso ao Ministro de Estado da Saúde.

O método empregado nesta pesquisa partiu da identificação, ano a ano, a contar de 2012 (data de publicação das primeiras recomendações da Conitec, constituída em 2011) até 2018, de todas as recomendações de deferimento ou não da incorporação de medicamentos ou protocolos clínicos. A partir daí, foram analisadas as recomendações em si, buscado verificar se tratavam doenças raras. No Brasil, a conceituação mais precisa sobreveio apenas com a publicação da Portaria no 199/2014, do Ministério da Saúde, que instituiu a Polícia Nacional de Atenção Integral às Pessoas com Doenças Raras e, no seu art. 3o, qualificou como rara "aquela que afeta até 65 pessoas em cada 100.000 indivíduos, ou seja, 1,3 pessoa para cada 2000 indivíduos" (BRASIL, 2014, documento online).

Em seguida, nas aludidas recomendações de indeferimento, foram identificadas, no tópico da discussão, a fundamentação determinante da conclusão e se houve menção, nos relatórios da Conitec, da excepcionalidade do medicamento em análise, principalmente a sua destinação a doenças raras, com baixa prevalência.

Por fim, as fundamentações determinantes foram tabeladas, ano a ano. As recomendações, no formato de relatórios, tomadas entre 2012 e 2018, estão disponíveis na internet, assim como as atas das reuniões da Conitec. Os dados foram tabulados na forma seguinte: 
TABELA 3 - Resultados da análise dos relatórios da CONITEC

\begin{tabular}{|c|c|c|c|c|}
\hline Relatório & $\begin{array}{l}\text { Medica- } \\
\text { mento }\end{array}$ & Doença & $\begin{array}{l}\text { Fundamento para não } \\
\text { incorporação }\end{array}$ & $\begin{array}{l}\text { Menção a } \\
\text { excepcionalidade da } \\
\text { doença }\end{array}$ \\
\hline $38 / 2013$ & $\begin{array}{l}\text { Ácido } \\
\text { ursodesoxi- } \\
\text { cólico }\end{array}$ & $\begin{array}{l}\text { Doença } \\
\text { hepatica } \\
\text { associada } \\
\text { à fibrose } \\
\text { cística }\end{array}$ & $\begin{array}{l}\text { Estudos com baixa } \\
\text { qualidade metodológica: } \\
\text { amostra com pequeno } \\
\text { número de pacientes, } \\
\text { grupos heterogêneos, } \\
\text { tempo de seguimento } \\
\text { curto, entre outros. }\end{array}$ & Não \\
\hline $76 / 2013$ & $\begin{array}{l}\text { Canaquinu- } \\
\text { mabe }\end{array}$ & $\begin{array}{l}\text { Síndromes } \\
\text { Periódicas } \\
\text { Associadas } \\
\text { à Criopirina }\end{array}$ & $\begin{array}{l}\text { Falta de estudos } \\
\text { comparativos e com maior } \\
\text { tempo de seguimento dos } \\
\text { pacientes resulta em } \\
\text { poucos subsídios para } \\
\text { fornecer uma adequada } \\
\text { avaliação dos benefícios e } \\
\text { riscos no uso do } \\
\text { medicamento em longo } \\
\text { prazo(...) }\end{array}$ & $\begin{array}{l}\text { Sim: o fato de se tratar de } \\
\text { uma doença rara, também } \\
\text { tem consequências na } \\
\text { avaliação econômica, que } \\
\text { apresentou todo o } \\
\text { seu modelo matemático } \\
\text { pautado em dados } \\
\text { extrapolados de outras } \\
\text { doenças, opinião de } \\
\text { especialistas e estimativas } \\
\text { para morte e expectativa } \\
\text { de vida. }\end{array}$ \\
\hline $129 / 2014$ & $\begin{array}{l}\text { Trióxido de } \\
\text { arsênio }\end{array}$ & $\begin{array}{l}\text { Leucemia } \\
\text { Promielocíti } \\
\text { ca Aguda } \\
\text { (LPA) }\end{array}$ & $\begin{array}{l}\text { A evidência encontrada } \\
\text { sobre eficácia e a } \\
\text { segurança é de baixa } \\
\text { qualidade. Os estudos } \\
\text { encontrados são de nível } \\
\text { metodológico baixo, não } \\
\text { randomizados, abertos e } \\
\text { não comparativos. }\end{array}$ & Não \\
\hline $163 / 2015$ & Icatibanto & $\begin{array}{l}\text { Crise } \\
\text { aguda } \\
\text { moderada } \\
\text { ou grave } \\
\text { do } \\
\text { Angioede- } \\
\text { ma } \\
\text { Hereditário }\end{array}$ & $\begin{array}{l}\text { Em relação aos estudos } \\
\text { disponíveis foram } \\
\text { encontradas evidências } \\
\text { limitadas, com população } \\
\text { pequena, heterogênea e } \\
\text { dispersa, tempo de } \\
\text { acompanhamento curto. }\end{array}$ & Não \\
\hline $348 / 2018$ & $\begin{array}{l}\text { Pegviso- } \\
\text { manto }\end{array}$ & $\begin{array}{l}\text { Acromega- } \\
\text { lia }\end{array}$ & $\begin{array}{l}\text { Evidências científicas, em } \\
\text { sua maioria, de baixa } \\
\text { qualidade metodológica. }\end{array}$ & Não \\
\hline
\end{tabular}

Fonte: Conitec (2012 - 2018).

Nos seis anos de atuação da Conitec, foram publicados cerca de 360 relatórios, e nesse conjunto, apenas cinco indeferimentos de incorporação com relação a doenças raras.O exame dos dados permite encaminhar a conclusão no sentido de que, no caso das doenças raras e medicamentos órfãos. 
Em apenas em um caso a excepcionalidade das doenças raras foi realmente levada em consideração, ainda que sem a necessária consequência de busca de uma regra intermediária ou de transição entre doenças com grande prevalência e doenças raras. A exceção se deu no relatório que tratou da Canaquinumabe, destinada ao tratamento da Síndromes Periódicas Associadas à Criopirina. Segundo o relatório, a raridade da doença "tem consequências na avaliação econômica, que apresentou todo o seu modelo matemático pautado em dados extrapolados de outras doenças, opinião de especialistas e estimativas para morte e expectativa de vida" (CONITEC, 2013, documento online). Ainda segundo o relatório,

\begin{abstract}
Essa é uma fragilidade prevista e reconhecida em estudos de doenças raras, porém, torna-se um obstáculo para a recomendação por parte dos sistemas de saúde pública, bem como para a avaliação do benefício frente aos tratamentos já disponibilizados, devido à fragilidade própria das estimativas apresentadas (CONITEC, 2013, documento online).
\end{abstract}

Com o objetivo de avaliar o impacto da rule of rescue nas recomendações de incorporação, foram analisados também todos os relatórios que resultaram na recomendação de incorporação de medicamentos órfãos, destinados a doenças raras, no mesmo período, assim identificados:

QUADRO 1 - Relatórios de Recomendação da CONITEC

\begin{tabular}{|c|c|}
\hline Relatório & Indicação \\
\hline Relatório 06 & Biotina para tratamento da deficiência de biotinidase. \\
\hline Relatório 293 & $\begin{array}{l}\text { Laronidase como terapia de reposição enzimática na } \\
\text { mucopolissacaridose tipo I. }\end{array}$ \\
\hline Relatório 311 & $\begin{array}{l}\text { Idursulfase como terapia de reposição enzimática na } \\
\text { mucopolissacaridose tipo II. }\end{array}$ \\
\hline Relatório 339 & $\begin{array}{l}\text { Tafamidis meglumina no tratamento da polineuropatia amiloidótica } \\
\text { familiar relacionada à proteína transtirretina. }\end{array}$ \\
\hline Relatório 19 & Acetato de lanreotida tratamento da acromegalia. \\
\hline Relatório 90 & $\begin{array}{l}\text { Ambrisentana e Bosentana para o tratamento da Hipertensão Arterial } \\
\text { Pulmonar na falha primária, secundária ou contra-indicação da } \\
\text { sildenafila. }\end{array}$ \\
\hline
\end{tabular}
Fonte: Conitec (2013).

Estes relatórios foram localizados a partir da consulta ao site da Conitec (INTERFARMA, 2018), por identificação das doenças raras e, posteriormente, 
pesquisa aos seus fundamentos, buscando as chaves "rule of rescue" ou "regra de resgate", alcançando-se igual constatação, no sentido de que esse conceito não foi considerado da mesma forma nas recomendações de incorporação. Da mesma forma, não há, em nenhuma das recomendações, menção à possibilidade de um tratamento excepcional, tal como a "rule of rescue", já admitida nos sistemas públicos de saúde do Reino Unido e Austrália.

\section{CONSIDERAÇÕES FINAIS}

A partir da análise realizada, e dos dados examinados, pode-se concluir que, até o presente momento, o conceito de rule of rescue, relacionado com a fixação de regras excepcionais para a incorporação de medicamentos órfãos aos sistemas públicos de saúde, ainda não exerceu uma influência determinante no direito brasileiro e nas recomendações da Conitec.

O relatório da Nice, mencionado no corpo do trabalho, dá conta de que aquele órgão já lhe conferia importância no ano de 2006, quando o Conselho de Cidadãos foi consultado a respeito de sua aplicação e limites.

No caso australiano, verifica-se que as deliberações do Australian Phamaceutical datam de $1995^{\circ}$, o que sinaliza para a urgência de que tal tema seja levado em consideração pela estrutura institucional brasileira.

Por fim, embora a ideia conceitual seja a mesma, o rule of rescue como exemplo de prioritarianismo, o certo é que compete a cada circunscrição nacional o estabelecimento dos seus próprios critérios, atendendo a necessidades específicas, o que pode levar a esquemas de decisão até mesmo diferentes. Exemplo desse contraste é a comparação entre as regras da Nice e do sistema público de saúde australiano: embora ambos aludindo a uma rule of rescue, os critérios de aplicação são divergentes e, em alguns aspetos, contraditórios.

\footnotetext{
${ }^{9}$ AUSTRÁLIA. Department of Health. Guidelines for the Pharmaceutical Industry on Preparation of Submissions to the Pharmaceutical Benefits Advisory Committee: including major submissions involving economic analyses. Canberra, 1995. Disponível em: $<$ http://www.health.gov.au/internet/wcms/ publishing.nst/Content/health-pbs-general-pubs-pharmpacgusubpac.htm>. Acesso em: 01/08/2018
} 


\section{REFERÊNCIAS}

ASSESSING the societal value in rare diseases by decision makers and patients. University Castilla-La Mancha (UCLM), Spain. AdvanceHTA - Rethinking the future of Health Technology Assessment. Conference, London, 10 nov. 2015. Disponível em: <http://www.advance-hta.eu/PDF/FinalConference/presentations/10Societal_Value_in_Rare_Diseases_UCLM.pdf>. Acesso em: 01 jul. 2018.

ASSOCIAÇÃO DA INDÚSTRIA FARMACÊUTICA DE PESQUISA. Doenças raras: a urgência do acesso à saúde. Interfarma, fev. 2018. Disponível em: <https://www. interfarma.org.br/public/files/biblioteca/doencas-raras--a-urgencia-do-acesso-asaude-interfarma.pdf>. Acesso em: 14 jun. 2018.

BRASIL. Decreto no 7.646, de 21 de dezembro de 2011. Disponível em: <http://www.planalto.gov.br/ccivil_03/_ato2011-2014/2011/decreto/d7646.htm>. Acesso em: 14 jun. 2018.

BRASIL. Ministério da Educação. Plataforma Sucupira. 01 abr. 2014. Disponível em: <http://www.capes.gov.br/avaliacao/plataforma-sucupira>. Acesso em: 14 jun. 2018.

BRASIL. Ministério da Saúde. Portaria no 199, de 30 de janeiro de 2014. Disponível em: <http://bvsms.saude.gov.br/bvs/saudelegis/gm/2014/prt0199_30_01_2014.html>. Acesso em: 14 jun. 2018.

BRASIL. Ministério da Saúde. Relatório de Recomendação da Comissão Nacional de Incorporação de Tecnologias no SUS. Canaquinumabe - Síndromes Periódicas Associadas à Criopirina (CAPS). Conitec, out. 2013. Disponível em: <http://conitec. gov.br/images/Incorporados/Canaquinumabe-final.pdf>. Acesso em 31 mai. 2018.

BRASIL. Ministério Público Federal. Ação do MPF requer elaboração de protocolos clínicos para doenças raras. Procuradoria da República no Distrito Federal, 14 mai. 2018. Disponível em: <http://www.mpf.mp.br/df/sala-de-imprensa/noticiasdf/acao-do-mpf-df-requer-elaboracao-de-protocolos-clinicos-para-doencas-raras $>$. Acesso em: 14 jun. 2018.

CIARLINI, Álvaro Luiz Araújo Sales. Análise jurídica dos critérios axiológicos de avaliação de medicamentos pela Comissão Nacional de Incorporação de Tecnologias ao SUS - Conitec. Cad. Ibero-Amer. Dir. Sanit., v. 5, n. 1. Brasília, 2016.

COOKSON, R.; MCCABE, C; TSUCHIYA, A. Public health care resource allocation and the rule of rescue. Health Economics and Decision Science Discussion Paper Series, n. 07/04. The University of Sheffield, 2007. Disponível em: <http://eprints. whiterose.ac.uk/10915/1/HEDS_DP_07-04.pdf>. Acesso em: 14 jun. 2018.

DIAS, Eduardo Rocha; SILVA JÚNIOR, Geraldo Bezerra da. A medicina baseada em evidências na jurisprudência relativa ao direito à saúde. Revista Einstein, v. 14, n. 1, 
jan./mar. 2016, pp. 1-5.

DUARTE, Clarice Seixas; BRAGA, Paulo Vitor Bérgamo. A utilização dos protocolos clínicos e diretrizes terapêuticas e a racionalização da judicialização do direito à saúde. Revista de Direito Sanitário, v. 18, n. 1, pp. 171-190, São Paulo, mar/jun. 2017.

FUNDAÇÃO OSWALDO CRUZ. Cadernos Ibero-Americanos de Direito Sanitário. Brasília-DF. Disponível em: <https://www.cadernos.prodisa.fiocruz.br/index.php/ cadernos/about/editorialPolicies\#focusAndScope>. Acesso em: 14 jun. 2018.

GOMES, Luanna de Mendonça. A saúde como objeto de consumo: uma análise sobre as demandas e consultas públicas de incorporação de medicamentos no SUS. Cad. Ibero-Amer. Dir. Sanit., v. 4, n. 4. Brasília, 2015.

HTA and rare diseases: assessing the societal value of orphan drugs. AdvanceHTA - Rethinking the future of Health Technology Assessment. Disponível em: <http://www.advance-hta.eu/wp3.php>. Acesso em: 01 jul. 2018.

HUGHES, D.A; TUNNAGE, B.; YEO, S.T. Drugs for exceptionally rare diseases: do they deserve special status for funding? QJM: An International Journal of Medicine, v. 98, ano 11, pp. 829-836, 01 nov. 2005. Disponível em: <https://academic.oup.com/ qjmed/article/98/11/829/1522301 >. Acesso em: 01 jul. 2018.

HUYARD, C. How did uncommon disorders become 'rare diseases'? History of a boundary object. Sociology of Health and IIIness, v.31, n.4, pp.463-477, 2009.

JONSEN, Albert R. Bentham in a Box: technology assessment and health care allocation. The Journal of Law, Medicine \& Ethics, set. 1986. Disponível em: $<$ http://journals.sagepub.com/doi/10.1111/j.1748-720X.1986.tb00974.x>. Acesso em: 12 jun. 2018.

KYMLICKA, Will. Filosofia política contemporânea. São Paulo: Martins Fontes, 2006.

LLINARES, J. A regulatory overview about rare diseases. In: PAZ, M. P. e GROFT, S. C. (Ed.) Rare diseases epidemiology. 1. ed. New York: Springer, 2010. cap. 12, pp. 193-207. (Advances in experimental medicine biology).

MCKIE, John; RICHARDSON, Jeff R. J. The rule of rescue. Social Science and Medicine, v. 56, ano 12, pp. 2407-2419, jul. 2003. Disponível em: <https://research. monash.edu/en/publications/the-rule-of-rescues. Acesso em: 01 jul. 2018.

NAGEL, Thomas. Mortal question. Cambridge: Cambridge University Press, 1979, pp. 123-124, apud PARFIT, Derek. Equality and priority. v. 10, n. 3. Oxford: Blackwekk Publishers, 1997. pp. 201-221. Disponível em: $<$ http://individual.utoronto.ca/stafforini/ parfit/parfit_-_equality_and_priority.pdf>. Acesso em: 13 jun. 2018. 
NATIONAL INSTITUTE FOR HEALTH AND CLINICAL EXCELLENCE. Citizens council report: the rule of rescue. Nice: London, 2006. Disponível em: $<$ https://www.nice.org.uk/Media/Default/Get-involved/CitizensCouncil/Reports/CCReport06RuleOfRescue.pdf>. Acesso em: 14 jun. 2018.

NITA, M.E.; NOBRE, M. R. C.; COSTA, A. M. N. Busca da evidência a partir do cenário clínico. In: NITA, M. E. et al (ed). Avaliação de Tecnologias em Saúde: evidência clínica, análise econômica e análise de decisão. cap. 10. Porto Alegre: Artmed, 2010. pp. 147-59.

NOGUEIRA, Karina Pires; CAMARGO, Erika Barbosa. Judicialização da saúde: gastos federais para o Sistema Único de Saúde (SUS) entre 2011-2014. Cad. IberoAmer. Dir. Sanit., v. 6, n. 2, pp. 120-132. Brasília, abr./jun. 2017.

NUSSBAUM, Martha. 'The central capabilities'. In: Creating Capabilities: The Human Development Approach. Cambridge MA: Belknap Press of Harvard University Press, 2011. pp. 17-45.

OLIVEIRA, C.R.C. et al. Políticas da 'expertise': doenças raras e gestão das tecnologias em saúde pelo SUS. Revista Brasileira de Ciência, Tecnologia e Sociedade, São Paulo, v.2, n.2, pp.150-186, jul./dez., 2011. Disponível em: <https://www.arca.fiocruz.br/handle/icict/6439>. Acesso em: 10 mai. 2018.

OLIVEIRA, Cláudio Cordovil; GUIMARÃES, Maria Cristina Soares; MACHADO, Rejane. Doenças raras como categoria de classificação emergente: o caso brasileiro. Revista de Informação, v. 13, n.1 fev. 2012, artigo 4.

PARFIT, Derek. Equality and priority. v. 10, n. 3. Oxford: Blackwekk Publishers, 1997. pp. 201-221. Disponível em:

$<$ http://individual.utoronto.ca/stafforini/parfit/parfit_-_equality_and_priority.pdf>. Acesso em: 13 jun. 2018.

SEN, AMARTYA. Sobre Ética e Economia. São Paulo: Companhia das Letras, 1999.

SMART, J.C.C.; WILLIAMS, B. Utilitarianism: for \& against. Cambridge: Cambridge University Press, 1973.

SOUZA, Marcelle de. Gasto com 10 remédios mais pedidos na Justiça para o SUS é de quase $\mathrm{R} \$ 1$ bi. Ministério da Saúde. Portal Arquivos. Disponível em:

<http://portalarquivos.saude.gov.br/images/pdf/2017/maio/15/06042017_Gasto-com10-remedios-mais-pedidos-na-Justica.pdf>. Acesso em: 01 jul. 2018.

UNIVERSIDADE DE SÃO PAULO. Revista de Direito Sanitário. São Paulo.

Disponível em: <https://www.revistas.usp.br/rdisan/about>. Acesso em: 14 jun. 2018.

WALDRON, Jeremy. Law and Disagreement. Grã-Bretanha: Oxford UK Pod, 2001.

WÄSTFELT, M.; FADEEL, B.; HENTER, J.I. A journey of hope: lessons learned from studies on rare diseases and orphan drugs. Journal of Internal Medicine, v. 260, 
p.10, 2006.

WOLFF, Jonathan; DE-SHALIT, Avner. Priority to the least advantaged. In: Disadvantage. Edited by Will Kymlicka and David Miller. New York: Oxford University Press, 2007. pp. 155-166.

Recebido em 15/08/2018

Aprovado em 19/12/2018

Received in 15/08/2018

Approved in 19/12/2018 